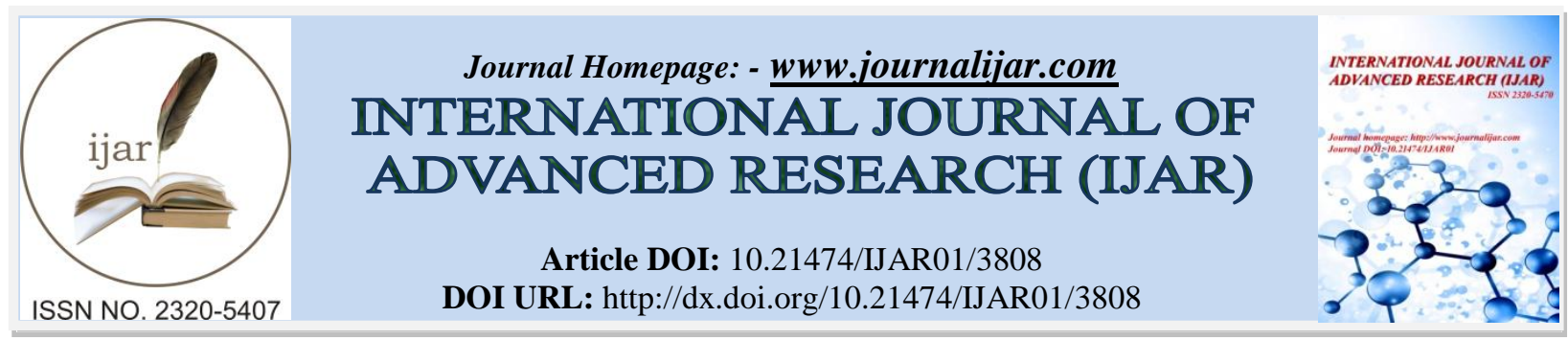

RESEARCH ARTICLE

\title{
DISSEMINATED NOCARDIOSIS DUE TO NOCARDIA SPECIES.
}

\section{Guliko Kiliptari $^{1}$, Tamar Didbaridze ${ }^{2}$, Nino Gogokhia ${ }^{3}$ and Khatia Mikaberidze ${ }^{4}$.}

1. Head of Department of critical care MD, PhD. TSMU the First University Clinic (Tbilisi, Georgia).

2. Microbiologist. MD. PhD.TSMU the First University Clinic (Tbilisi, Georgia).

3. Head of Clinical Laboratory. MD. PhD.TSMU the First University Clinic (Tbilisi, Georgia).

4. Laboratory Phisician, TSMU the First University Clinic(Tbilissi, Georgia).

\section{Manuscript Info}

\section{Manuscript History}

Received: 01 February 2017

Final Accepted: 01 March 2017

Published: April 2017

Key words:-

Nocardiaspp, blanching rod, Nocardiosis

\begin{abstract}
Nocardiosis is a vital, but often ignored, infectious disease in immunocompromised hosts, which is particularly serious in the absence of timely diagnosis and therapy. Nocardiosis affects patients who display a cellular immunodeficiency, such as transplant recipients on immunosuppressive treatment, but uncommonly associated with high morbidity and mortality rates. Disseminated Nocardiosis affecting the central nervous system (CNS), abdomen, skin and lungs .Nocardia spp. infection can be observed and confirmed by subculture and positive microscopic detection of a branching gram-positive rod.

Infection commonly enters via the respiratory route but may also occur through skin trauma. A case of disseminated nocardiosis in an immunocompetent individual after car accident is presented. The case highlights challenges surrounding diagnosis and microbiological identification of Gram-positive branching bacilli, patient management, and choice of antibiotic.
\end{abstract}

Copy Right, IJAR, 2017,. All rights reserved.

\section{Introduction:-}

Nocardiosis is rare infection and caused by nocardiaspecies, from family of actinomycetes. Over 100species of the genus Nocardia have been identified.most infection are due from Nocardia asteroides complex, includes heterogeneous group of organisms. N.brasilines is associated with tropical environments, The first case of N.brasiliensis in Europe has been described in 1968. N.brasiliensisismorefrequentlyrecoveredfromsoil .Nocardiaorganismsisgram - positivebacteria Nocardiosis is an acute,subacute or chronic infectious disease,has local or disseminated character. Desease occurs in cutaneous,pulmonary, brain,skin forms. Primary cutaneous nocardiosis is higher and are ubiquitous in the soil $(1,2,3)$.

Cutaneous nocardiosis presents as cutaneous(cellulitis ,abscess),lymphocutaneous (sporotrichoid nocardiosis),subcutaneous infections.Cutaneousnocardiosis also manifests as multiple erythematous nodules after traumatic injury $(5,6)$. The illness has disseminated character in immonocompromised patients , with vasculits,lupuserithematousis, chronic renal failure, after transplantation and steroids treatment. Pulmonary infections may lead empiema and abscess.CNS infection manifests as abscess with meningitis or without meningitis. Nocardia infection is usually slow to respond to treatment, it can be life-threatening for those with weakened immune systems, especially if diagnosis and treatment are delayed. It's important to seek treatment as soon as symptoms develop. This can help prevent the infection from spreading and causing complications $(7,8)$.

Corresponding Author:- Guliko Kiliptari.

Address:- Head of Department of critical care MD, PhD. TSMU the First University Clinic (Tbilisi, Georgia). 


\section{Case report:-}

A32 year old man was admitted to the intensive care unit(the First University Clinic of TSMU, Tbilisi,Georgia) after car accident.

Diagnosis:polytrauma,head closed trauma ,brain contusion, acute subarachnoid haemorrhage,scalped wound in temporal and parietal area. Closed chest trauma, lung contusion, fracture of shoulder bone, multiple and open fracture of shin bone. Excoriation of chest,abdomen,pelvic ,both extremities area,multiplesubcutaneous hematomas.At admission patient was in coma (GCS 4-5),hemodynamia was unstable and was used norepinephrin infusion .In operating room have

been performed scalped wound surgical treatment and left shin osteosinthesis.

Chest CT revealed bilateral lung contusion. From second day of admission,--- hyperthermia >39$40^{\circ} \mathrm{C}$, leicocytosis $\left(20 \times 10^{9} / \mathrm{l}\right)$ and rash on full body surface. On chest second CT scan revealed bilateral ,dorsal infiltration in lung parenchyma.(picture1).

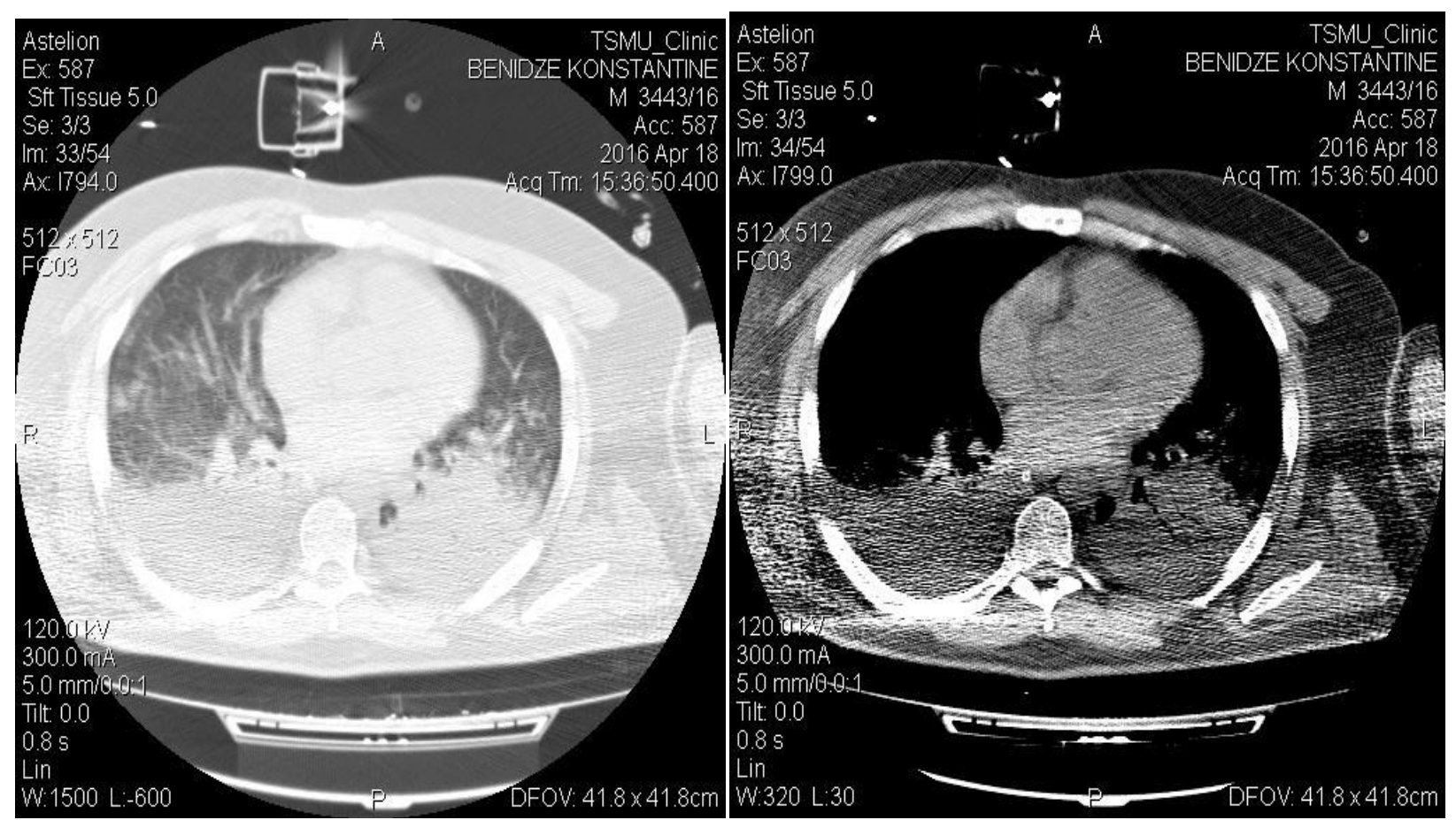

Picture1:- Antibacterial treatment --piperacillin/tazobactam,moxifloxacin.Respiratory secretion was sent for bacteriologicalanalisis. On seven day after admission was identified Nocardia $\mathbf{s p p . 1 0} / \mathbf{m l}$ in sputum.Blood culture analisis revealed Staphylococcus aureus $10^{8} / \mathrm{ml}$.By bacteriologicalanalisis of CSF bacterial growth was not determined. 


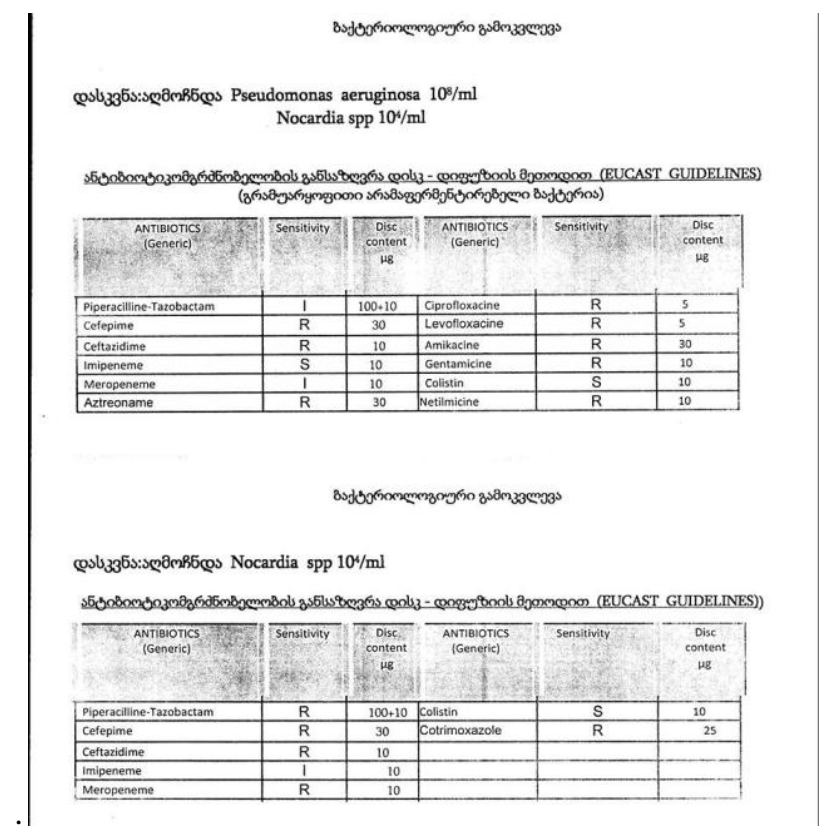

Picture2:- On twentieth day after admission in tracheal aspirate again was identified Nocardia $\mathbf{s p p . 1 0} \%$ ml.

Patient was on mechanical ventilation. Soft tissue ultrasound detects liquid area in upper, lateral part of boththigh.Bilateralphlegmon was drained and aspirate was sent for bacteriological investigation .In culture was

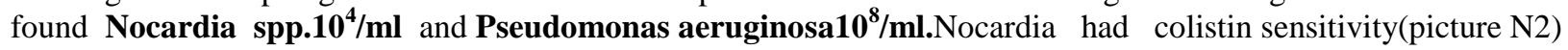

Respiratory secretion(sputum) and aspirates from abscesses was taken and sent under compliance with the appropriate protocol for routine culture and sensitivity test.The bacteriological research included: isolation of a pure culture, Gram staining, use of the rapid identification systems (api20E,api Staph, api Strep, api A, api20Caux, biomerieux) and Antimicrobial Susceptibility Testing (AST) determination through Kirby-Bauer method by using of standard discs (EUCAST guidelines). Sputum and aspirates from abscess was cultured in aerobic atmosphere on the enrichment and differential-diagnostic medium : TSA 5\% sheep blood, Chocolate agar, Endo agar and Sabouraud dextrose agar.After 48 hour of incubation at $37^{\circ} \mathrm{C}$, appeared colony, which were stained by use of Gram procedure.The isolate was gram-positive, bacillary, branching filamentous bacteria whose hyphae was fragment to coccobacillary forms. Originally, identification of the nocardial species was based on hydrolysis of casein, tyrosine, xanthine, and hypoxanthine and by the amplification profile index special panel (api20C AUX) for fungy. Blood culture were negative for Nocardia organisms, which is positive in minority of patients, but they always should be obtained when pulmonary or disseminated nocardiosis is suspected.

Patient with pulmonary nocardiosis presented with findings of pulmonary consolidation, cutaneousnocardiosis with phlegmon in soft tissue. Cutaneous and soft tissue nocardiosis was result from traumatic injury to the skin that involved contamimnation with soil.

Polymicrobalbloodstream and respiratory tract infection had also been identified. The combination of pneumonia and lower extremity abcess is suggestive of disseminated nocardiosis.Respiratory secretions, skin biopsy samples and aspirates from abscesses was common specimens from wichNocardia species was identified .The patient was discharged from hospital with the improved condicion and proper recommendacion.

\section{Conclusion:-}

In a case of 32 years old man, the diagnosis of disseminated Nocardiosis was established by sputum and aspirates from abscess microbiology. The case highlights challenges surrounding diagnosis and microbiological identification of Gram-positive branching bacilli, patient management, and choice of antibiotic.Diagnosis should be presumed early and microbiological conditions should be optimized, in order to identify the species and achieve antibiotic susceptibility testing. This is a very important step to choose an effective therapeutic regimen or alternative options. 


\section{Reference:-}

1. McNeil M.M., Brown J.M. The medically important aerobic actinomycetes: epidemiology and microbiology. ClinMicrobiol Rev. 1994;7(3):357-417

2. Young L.S., Rubin R.H. Mycobacterial and nocardial diseases in the compromised host. In: Rubin R.H., Young L.S., editors. A Clinical Approach to Infection in the Compromised Host. 4th ed. Kluwer Academic; New York, NY: 2002. pp. 257-261.

3. Martinez R., Reyes S., Menendez R. Pulmonary nocardiosis: risk factors, clinical features, diagnosis and prognosis. CurrOpinPulm Med. 2008;14(3):219-227. [PubMed]

4. Kontoyiannis D.P., Jacobson K.L., Whimbey E.E., Rolston K.V., Raad I.I. Central venous catheterassociated Nocardia bacteremia: an unusual manifestation of nocardiosis. Clin Infect Dis. 2000;31(2):617618. [PubMed]

5. Munoz J., Mirelis B., Aragon L.M. Clinical and microbiological features of nocardiosis 1997-2003. J Med Microbiol. 2007;56(pt 4):545-550. [PubMed]

6. Gombert M.E., Berkowitz L.B., Aulicino T.M., duBouchet L. Therapy of pulmonary nocardiosis in immunocompromised mice. Antimicrob Agents Chemother. 1990;34(9):1766-1768. [PMC free article][PubMed]

7. McNeil M.M., Brown J.M., Hutwagner L.C., Schiff T.A. Evaluation of therapy for Nocardiaasteroides complex infecton: CDN/NCID report. Infect Dis ClinPract. 1995;4(4):287-292.

8. McNeil M.M., Brown J.M. The medically important aerobic actinomycetes: epidemiology and microbiology. ClinMicrobiol Rev. 1994;7(3):357-417 